Degeneration of the fibers in the brain was studied by Nauta method on an autopsy case of intractable pain due to cancer of tongue who died by aggravation of the cancer, 13 months after the very effective thalamolaminatory. Degenerative fibers were seen in the Vcpc nucleus of Hassler which was thought to be closely related to pain mechanisms. Degeneration was found also in the second somatosensory cortex.

From these findings, the authors consider that the internal medullary amina is not a structure of even constitution, but there may be functional topographical differences, namely, anterior, middle and posterior portions are concerned with $\in$ motional, motor and sensory functions respectively.

\title{
7. Studies on the Function of Pulviuar
}

\author{
Tatsuyuki Kudo, Nobuo Yoshir, Kimihiro Minakami, \\ Toru MiNe and Kazumasa ADACHI \\ Dept. of Neurosurgery, Keio University \\ Shiro SHIMIZU \\ Dept. of Neurosurgery, Tokyo Musashino Hospital
}

Hiroshi Nakahama, Sadao Aikawwa and Nobuko Nishioka

Seishin Igaku Kenkyujo

\section{Neurophysiological Investigation of the Midbrain Tegmentum in Man}

\author{
Hiromu Hasegawa, Osamu Nakai, Kozo Yajima, Setsuo Nakajo, \\ Osamu Seino, Isamu EzuKa and Komei UeKI \\ Department of Neurosurgery, Brain Research Institute, Niigata University
}

Involuntary movement is supposed to be induced by the pathological state of the basal ganglia, red nucleus and nigral nucleus, etc. Among these, the physiological study of animal red nucleus is fairly advanced and the role it plays in the muscle tonus adjustment is already proved. Human red nucleus, however, is different in its anatomical structure and its function is still obscure.

We have investigated the functional role played by human red nucleus by inserting a stimulating electrode into the midbrain tegmentum during stereotaxic thalamotomy. At the same time the possibility of correcting radiological target by physiological device is also examined.

Evoked potential observed on the scalp or on the epidural EEG is shown in well-known form when a stimulating electrode is in the thalamus but generally 